\title{
Utility of Telomerase-pot1 Fusion Protein in Vascular Tissue Engineering
}

\author{
Thomas H. Petersen, ${ }^{*} \dagger$ Thomas Hitchcock, $\uparrow$ Akihito Muto, $\dagger$ Elizabeth A. Calle, $\dagger$ \\ Liping Zhao, $\uparrow$ Zhaodi Gong, $\dagger$ Liqiong Gui, $\uparrow$ Alan Dardik, $\dagger$ Dawn E. Bowles, $\neq$ \\ Christopher M. Counter, $\S$ and Laura E. Niklason $\dagger$ \\ *Department of Biomedical Engineering, Duke University, Durham, NC, USA \\ $\nmid$ Program in Vascular Biology and Therapeutics, Yale University, New Haven, CT, USA \\ ‡Department of Surgery, Duke University, Durham, NC, USA \\ $\S$ Department of Pharmacology and Cancer Biology, Duke University, Durham, NC, USA
}

\begin{abstract}
While advances in regenerative medicine and vascular tissue engineering have been substantial in recent years, important stumbling blocks remain. In particular, the limited life span of differentiated cells that are harvested from elderly human donors is an important limitation in many areas of regenerative medicine. Recently, a mutant of the human telomerase reverse transcriptase enzyme (TERT) was described, which is highly processive and elongates telomeres more rapidly than conventional telomerase. This mutant, called pot1-TERT, is a chimeric fusion between the DNA binding protein pot1 and TERT. Because pot1-TERT is highly processive, it is possible that transient delivery of this transgene to cells that are utilized in regenerative medicine applications may elongate telomeres and extend cellular life span while avoiding risks that are associated with retroviral or lentiviral vectors. In the present study, adenoviral delivery of pot1-TERT resulted in transient reconstitution of telomerase activity in human smooth muscle cells, as demonstrated by telomeric repeat amplification protocol (TRAP). In addition, human engineered vessels that were cultured using pot1-TERT-expressing cells had greater collagen content and somewhat better performance in vivo than control grafts. Hence, transient delivery of pot1-TERT to elderly human cells may be useful for increasing cellular life span and improving the functional characteristics of resultant tissue-engineered constructs.
\end{abstract}

Key words: Telomerase; Senescence; Tissue engineering; Vascular grafts; Smooth muscle cells

\section{INTRODUCTION}

Vascular disease is a pressing problem in today's society, creating a strong demand for small-diameter vascular bypass conduits. Autologous venous grafts are often used, although approximately $12 \%$ of all patients suffering from coronary artery disease are ineligible for current methods of surgical intervention $(18,20)$. There is additional need for small-diameter vascular grafts for patients requiring dialysis access and those suffering from peripheral vascular disease. This work is targeted at the eventual development of engineered small-diameter vascular grafts, using a patient's own cells as the source.

Previous work from our laboratory has demonstrated the feasibility of growing engineered vascular grafts using human cell sources (15). However, patients who are in need of a replacement vascular conduit tend to be elderly or suffer from vascular disease, meaning that autologous cell sources are frequently inadequate due to shortened replicative life span (16). In order to create an engineered vessel, extensive cellular expansion is required in the laboratory, and the cells from elderly patients are often not capable of sufficient expansion to allow growth of a functional vessel (15). In order to circumvent this problem, we and others have utilized the reverse transcriptase telomerase to extend telomeres and cellular replicative capacity $(4,21,25)$.

Telomerase consists of an RNA primer subunit and an enzymatic subunit. Telomerase reverse transcriptase (TERT) is the enzymatic component of human telomerase, which is coupled with an RNA primer in vivo to provide telomere-extending capability. Although the RNA component of telomerase is expressed in most cells, TERT is only expressed in gametes, cells of the fetus, and in adult stem cells, although some cell types

Received January 19, 2009; final acceptance October 14, 2009. Online prepub date: October 29, 2009.

Address correspondence to Laura E Niklason, M.D., Ph.D., Vice-Chair of Anesthesiology, Professor of Anesthesiology and Biomedical Engineering, Yale University, Room 301D Amistad Building, 10 Amistad Street, New Haven, CT 06519, USA. Tel: 203-737-1422; Fax: 203-737-1484; E-mail: laura.niklason@yale.edu 
can express low levels of telomerase activity during life. Studies in our laboratory have shown that introduction of ectopic TERT in vascular cells from elderly donors expands cellular life span and allows for the culture of engineered vessels from patients up to 74 years old (21). This extension of telomere length, and consequent extension of replicative life span, was effected over many population doublings, necessitating a retroviral vector to confer permanent TERT expression. However, there are concerns regarding the use of TERT and retroviruses in cells that are destined for human implantation. Although telomerase is not an oncogene, it is activated in many types of cancer (24) and concern exists that TERT activation could increase the probability of carcinogenic transformation in an implanted tissue. In addition, retroviral delivery of TERT or any other gene may increase the risk of cancer, in light of previous results of gene therapy trials for severe combined immunodeficiency (8).

The DNA-binding protein protection of telomeres (pot1) is the principle single-stranded DNA binding protein at the telomere $(3,5)$. Together with a host of other proteins, pot1 forms the complex termed shelterin (17), which regulates telomerase activity at the telomere and which protects the single-stranded telomere end from DNA repair mechanisms $(12,13)$. Earlier work has shown that fusion of pot1 to TERT allows the rapid extension of telomeres $(1,5)$. The ability of the pot1-TERT fusion protein to rapidly lengthen telomeres gives rise to the idea that this fusion protein could be used to transiently and rapidly lengthen telomeres in cells that were destined for cellular therapy. In other words, we hypothesized that transient ectopic expression of pot1-hTERT in elderly cells would lengthen telomeres sufficiently to increase cellular life span and render these cells useful for cellular therapies. In addition, avoidance of any retroviral vector, and strictly transient delivery of the transgene, may enhance the safety of cell life span extension for regenerative medicine.

In order to investigate this hypothesis with regard to the development of an engineered vessel, we transduced human smooth muscle cells from a single adult human donor with a pot1-TERT-encoding adenovirus. The cells were studied in vitro for telomerase activity and for cellular replicative potential. Subsequently, we grew smalldiameter vessels using both the pot1-TERT cells and control cells in specially designed bioreactors. The vessels were assayed for collagen content, apoptosis, and cellular proliferation. A small number of vessels were implanted into nude rats as abdominal aortic interpositional grafts. We found increased life span and telomerase activity in vitro and increased turnover in vessel culture of the pot1-TERT-transduced cells, as well as increased collagen deposition, compared to control and GFP-transduced cells. In addition, implanted vessels from the pot1-TERT cell population performed better than control vessels. Together, these results suggest that transient activation of telomerase may be promising for tissue engineering applications using cells from an elderly donor.

\section{MATERIALS AND METHODS}

\section{Plasmids, Viruses}

The pot1-TERT fusion construct was engineered into the pBabe-hygro plasmid. A pBabe-GFP-hygro plasmid, with green fluorescent protein (GFP) in place of pot1TERT, was used as a vector control. Replication incompetent adenoviral vectors expressing the pot1-TERT transgene were generated using the pAdeasy system according to the supplier's directions (Stratagene). Briefly, the pot1-TERT coding regions were removed from the pot1-TERT pBabe plasmid using the NheI and SalI restriction sites. This fragment was cloned into the pCMV shuttle plasmid between the BglII and SalI restriction sites. This CMV-pot1-TERT shuttle was recombined with the AdEasy vector in BJ5183 E. coli cells to generate the pot1-TERT Adeasy vector, which was transduced into HEK 293 cells and successively amplified. Recombinant pot1-TERT adenoviral vector was purified via ultracentrifugation on two successive cesium chloride gradients. Control GFP adenovirus was provided by Dawn Bowles. Plasmids were verified by PCR. Plasmid construction is demonstrated in Figure 1.

\section{Cell Isolation}

Human aortic smooth muscle cells (SMC) were isolated from a deceased 41-year-old male using standard explant techniques and with approval from the Yale University Human Investigation Committee. Isolated cells were expanded in DMEM containing $10 \%$ fetal bovine serum (Hyclone), 1\% penicillin/streptomycin (Sigma), and SmGM-2 aliquots (Lonza, Hopkinton, MA; the aliquots of antibiotic and serum from the SmGM-2 were not used). SMC were grown in humidified incubators at $37^{\circ} \mathrm{C}$ and $5 \% \mathrm{CO}_{2}$. Cells were either cultured without viral transduction or were transduced for $48 \mathrm{~h}$ with one of two adenoviruses: the pot1-TERTcontaining adenovirus, or a green fluorescent protein (GFP) control adenovirus. A population doubling (PD) value of 0 was arbitrarily assigned to the first confluent plate after infection.

\section{Engineered Vessel Culture}

Blood vessel bioreactors, pulsatile flow systems, and polyglycolic acid (PGA) meshes were prepared as described previously (19). PGA mesh was prepared around $1 \mathrm{~mm}$ outer diameter (OD) silicone tubing (St. Gobain Performance Plastics, Beaverton, MI) and seeded with either uninfected SMC, SMC infected with an adenovi- 


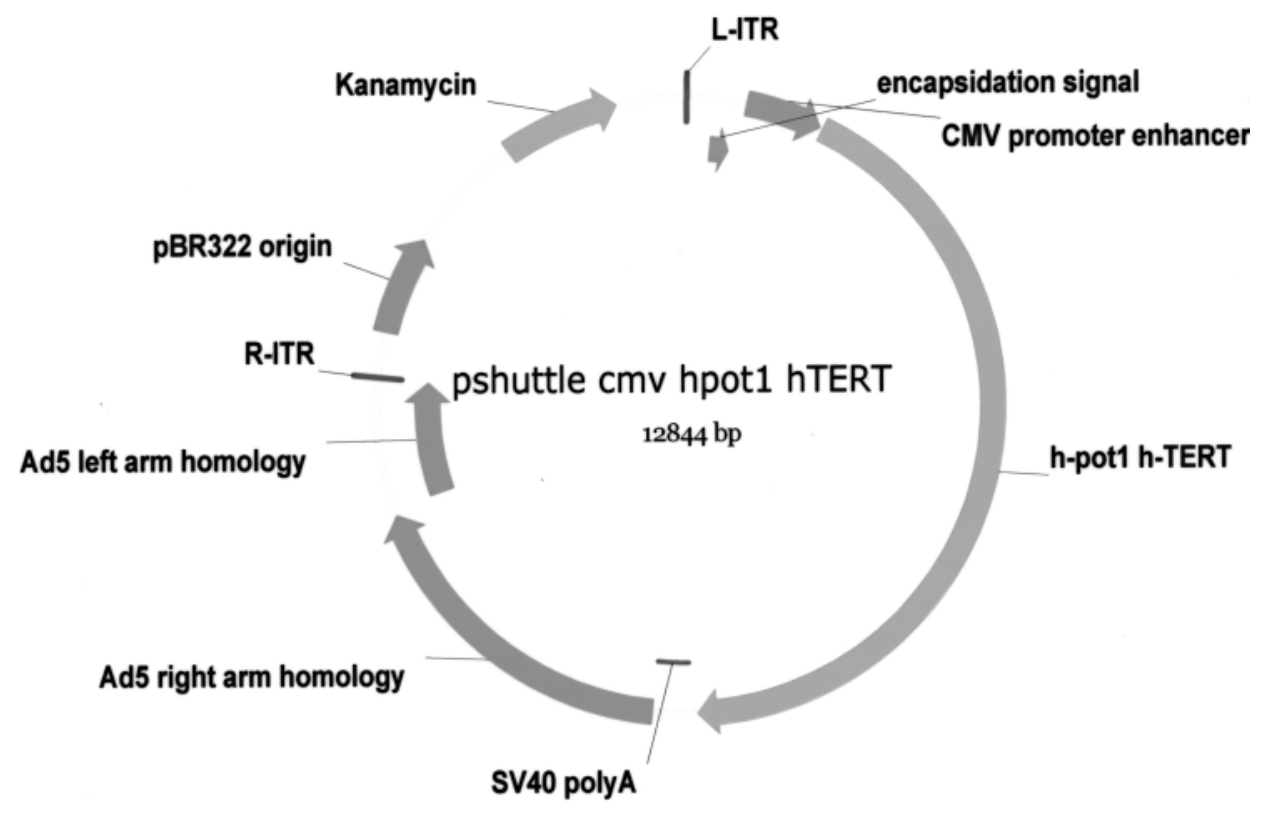

Figure 1. Plasmid map of pot1-TERT vector.

rus encoding pot1-TERT, or SMC infected with a vector control adenovirus encoding GFP. SMC were seeded onto the PGA mesh at $5 \times 10^{5}$ cells per mesh, and bioreactors were filled with culture medium, consisting of DMEM (Gibco) with $20 \%$ fetal bovine serum (Hyclone); $50 \mathrm{mg} / \mathrm{L}$ of ascorbic acid, proline, and glycine (Sigma); $20 \mathrm{mg} / \mathrm{L}$ alanine; $0.75 \mu \mathrm{g} / \mathrm{L}$ copper sulfate (Sigma); 10 mM HEPES (JRH Biosciences); and $10 \mathrm{ng} / \mathrm{ml}$ of basic fibroblast growth factor and platelet-derived growth factor B (Sigma). Engineered vessels were grown with pulsation at $\sim 165 \mathrm{bpm}$ and $\sim 1 \%$ radial distension for 8 weeks in humidified incubators at $37^{\circ} \mathrm{C}$ and $5 \% \mathrm{CO}_{2}$.

\section{Vessel Implantation}

All animal experiments were reviewed and approved by the Institutional Animal Care and Use Committee at Yale University. Male 3-month-old nude rats (CrI:NIHrnu) were purchased from Charles River Laboratories (Wilmington, MA). After induction of anesthesia, a midline laparotomy was performed and the visceral organs retracted to expose the abdominal aorta. The engineered vessels were implanted as aortic interpositional grafts using 10-0 prolene suture and standard operative techniques, and then the abdomen was closed. A single intraoperative dose of heparin was administered at $70 \mathrm{U} / \mathrm{kg}$ for anticoagulation. Necropsy was performed to retrieve the grafts for histological analysis.

\section{Telomerase Activity}

Lysates from the SMC were prepared in RIPA buffer (Boston Bioproducts) with added protease inhibitors
(Sigma) and assayed for telomerase activity using the TRAPeze Telomerase Detection Kit (Chemicon, Billerica, MA) according to the manufacturer's instructions.

\section{Collagen Analysis}

Collagen was quantified using a colorimetric assay to detect OH-proline using a modified Grant's method (7). Vessel samples were weighed and lyophilized, then incubated in papain $(140 \mu \mathrm{g} / \mathrm{ml})$ at $60^{\circ} \mathrm{C}$ overnight. Papain-digested samples were incubated in $6 \mathrm{~N} \mathrm{HCl}$ at $115^{\circ} \mathrm{C}$ for $18 \mathrm{~h}$, neutralized, oxidized with chloramine$\mathrm{T}$, and reacted with $p$-dimethylaminobenzaldehyde. Absorbance was measured at a wavelength of $550 \mathrm{~nm}$ and a 1:10 w/w ratio of hydroxyproline to collagen was used to calculate the collagen content of the vessels. Lyophilized vessel weights were used to determine percentage collagen content.

\section{Immunohistochemistry}

Vessel samples from before and after implantation were fixed in formalin, dehydrated, and embedded in paraffin. Thin $(5 \mu \mathrm{m})$ sections were deparaffinized and immunostained to assay for dividing and apoptotic cell nuclei. Cell proliferation was assessed via staining for proliferating cell nuclear antigen (PCNA) (Zymed, San Francisco, CA), and apoptotic nuclei were detected with terminal dUTP nick-end labeling (TUNEL) stain (Calbiochem, San Diego, CA). Sections were counterstained with hematoxylin or methyl green, and positive and negative nuclei were counted by a blinded researcher. Histo- 
logical stains for H\&E were performed by the Yale University Research Histology facility.

\section{RESULTS}

Pot1-TERT SMC Demonstrate Increased Telomerase Activity and Increased Cellular Replication

Control human SMC, isolated from adult proximal aorta, were infected with GFP-containing or pot1TERT-containing adenoviral constructs (Fig. 1). Cultures were split at passage 5 , with some being used for in vitro analysis and others being used to grow engineered vessels.

Telomerase activity was assessed using telomere repeat amplification protocol (TRAP) assay, shown in Figure $2 \mathrm{~A}$. The TRAP assay confirms the presence of functional telomerase activity in cell lysates. Functional telomerase results in $6 \mathrm{bp}$ laddering of the telomeric TTAGGG repeat. Absence of the laddering after heat inactivation of telomerase confirms that the 6-bp laddering is specific for telomerase activity and does not represent PCR artifact. The TRAP assay showed significant telomerase activity only in positive control cells (provided with the kit), as well as cells that were transduced with adenoviral pot1-TERT at passage 5 after transduction. The same pot1-TERT-infected cells at higher passage (passage 8) did not show telomerase activity on TRAP assay, implying that the vector and transgene were lost during serial passage. Cells that were transduced with GFP-containing adenovirus, and cells that were untransduced, did not show demonstrable telomerase activity (Fig. 2A).

Growth curves were obtained after transduction to examine the cellular life span for pot1-TERT-transduced and control cells (Fig. 2B). Growth of control cells reached a plateau by approximately 12 population doublings (PDs) after initial transduction, consistent with prior reports showing the inability of human SMC to proliferate extensively during in vitro culture (10). Cells that were transduced with the GFP adenovirus also slowed replication at the same time, confirming that the GFP-containing adenovirus did not affect cell proliferation either positively or negatively. In contrast, pot1TERT-transduced SMC continued to proliferate beyond this point. These results confirm the observations seen on TRAP assay regarding telomerase activity, and imply that telomerase activity in pot1-TERT cells resulted in some extension of replicative life span.

\section{Pot1-TERT SMC Demonstrate Increased Cellular Turnover in an Engineered Vessel}

Small-diameter $(1 \mathrm{~mm})$ vessels were grown in vitro in specially designed bioreactors, using the pot1-TERT cells, GFP-transduced cells, or nontransduced control

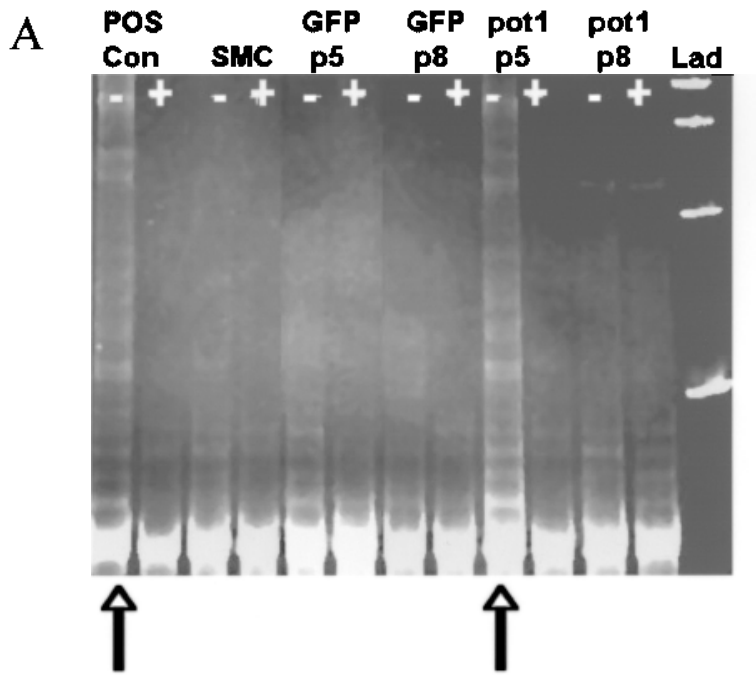

$\mathrm{B}$

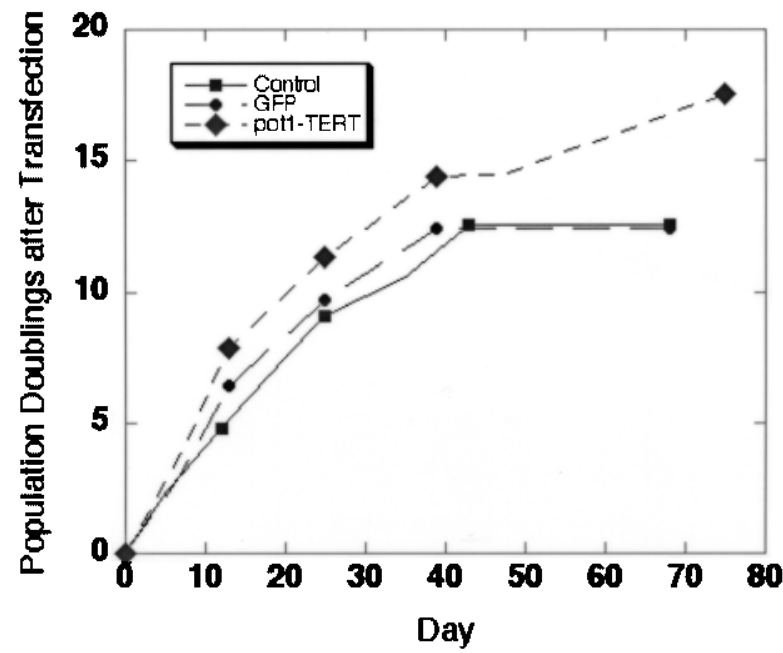

Figure 2. Telomerase activity and growth curves. (A) TRAP blot showing PCR reaction products from cellular lysates. Lane Pos Con: positive control H293 cells contained in TRA Peze kit; lane SMC: untransduced control SMC from a 41year-old donor; lanes GFP p5 and GFP p8: GFP-transduced cells at passage 5 and 8 , respectively; lanes pot 1 p5 and pot 1 p8: pot1-TERT-transduced cells at passage 5 and 8 , respectively; lane Lad: molecular weight ladder. (B) Growth curves for untransduced control cells (squares), GFP-transduced cells (circles), and pot1-TERT-transduced cells (diamonds).

cells. After 8 weeks of culture, resultant vessels were assessed for cell proliferation and apoptosis, collagen content, and histologic appearance (Fig. 3). Due to limitations in total cell number available from a single human donor, we were able to culture two vessels each from pot1-TERT cells, GFP-transduced cells, and control (untransduced) smooth muscle cells. Each vessel had a diameter of $1.0 \mathrm{~mm}$ and a length of $8 \mathrm{~cm}$. Vessels 

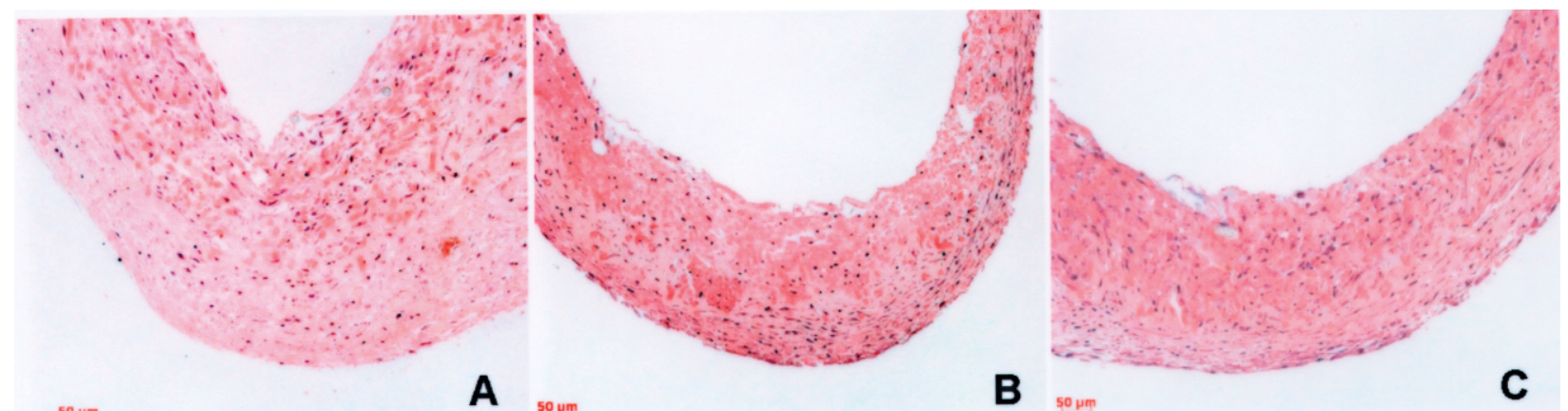

Figure 3. Histologies of human engineered vessels. Hematoxylin \& eosin staining of vessels cultured for 8 weeks. Vessels cultured from untransduced control SMC (A), GFP-transduced SMC (B), and pot1-TERT-transduced SMC (C). Scale bar: $50 \mu \mathrm{m}$.

grown from the untransduced control, GFP-transduced, and pot1-TERT-transduced cells had similar gross appearances on histology (H\&E staining) (Fig. 3).

Cell proliferation for each of the six vessels was assessed using staining for PCNA, and apoptosis was assessed using TUNEL. The two vessels grown from pot1TERT cells demonstrate many proliferating nuclei as well as apoptotic cell nuclei (Fig. 4). By contrast, the vessels cultured from control SMC and the GFP-transduced vessels showed fewer proliferating and apoptotic nuclei on multiple histological sections. In order to quantify the PCNA and TUNEL results, we stained several sections of each vessel and counted positive and negative nuclei as a percentage of total (Fig. 5). Pot1TERT vessels demonstrated $20 \% \mathrm{PCNA}^{+}$nuclei and $21 \% \mathrm{TUNEL}^{+}$nuclei, versus approximately $5 \% \mathrm{PCNA}^{+}$ and $3 \% \mathrm{TUNEL}^{+}$nuclei in the control SMC vessels. Data from GFP-transduced vessels were similar to the control SMC vessels. The increased cellular proliferation in vessels cultured from pot1-TERT cells likely reflects increased life span conferred by the transient activation of pot1-TERT, consistent with TRAP assay
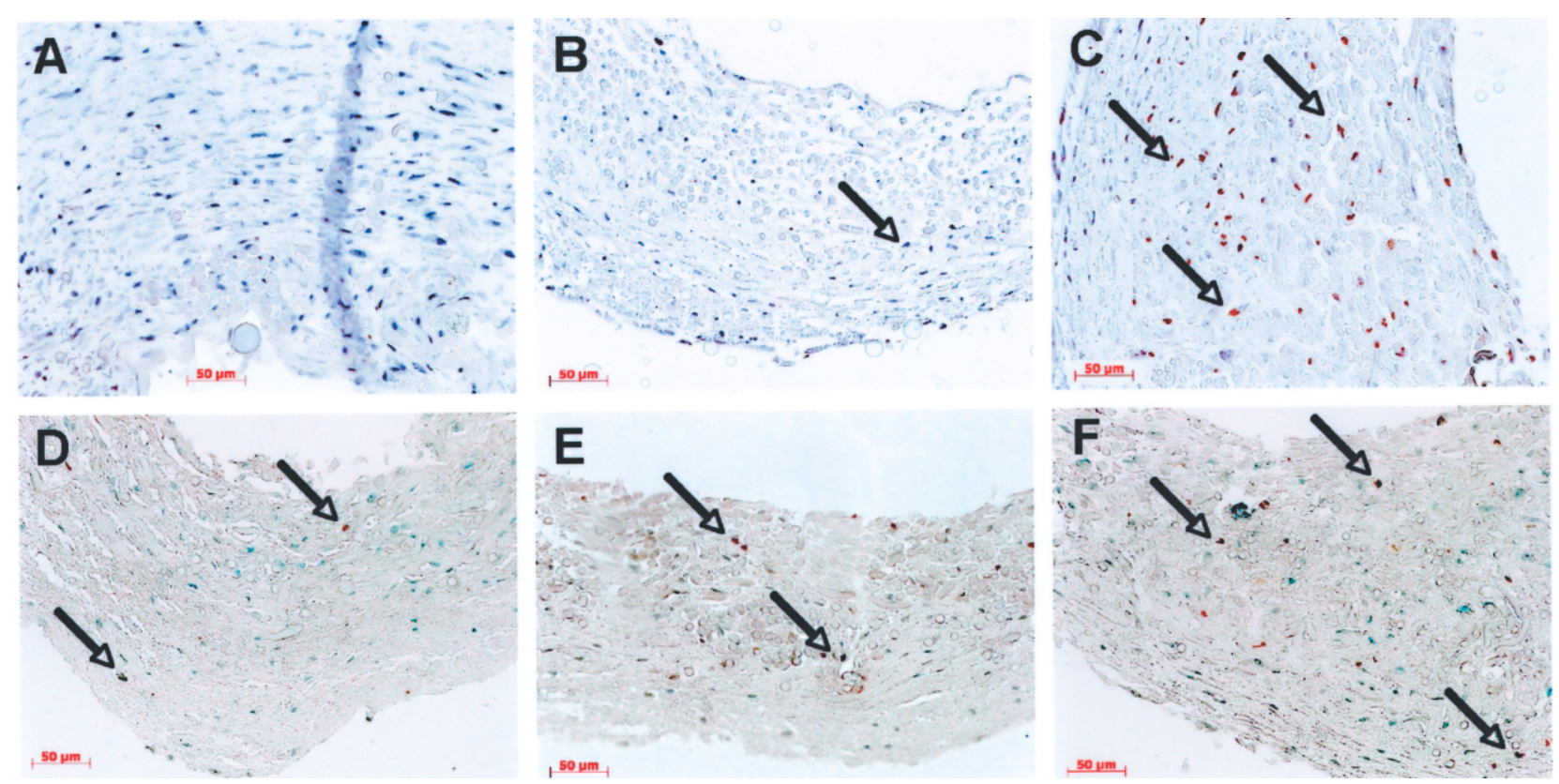

Figure 4. PCNA and TUNEL staining. PCNA staining of vessels cultured from untransduced control SMC (A), GFP-transduced SMC (B), and pot1-TERT-transduced SMC (C). Positive nuclei stain brown, negative nuclei stain purple with hematoxylin counterstain. TUNEL staining of vessels cultured from untransduced control SMC (D), GFP-transduced SMC (E), and pot1-TERT-transduced SMC (F). Positive nuclei stain brown, negative nuclei stain green with methyl green counterstain. Arrows indicate positive nuclei. Scale bar: $50 \mu \mathrm{m}$. 


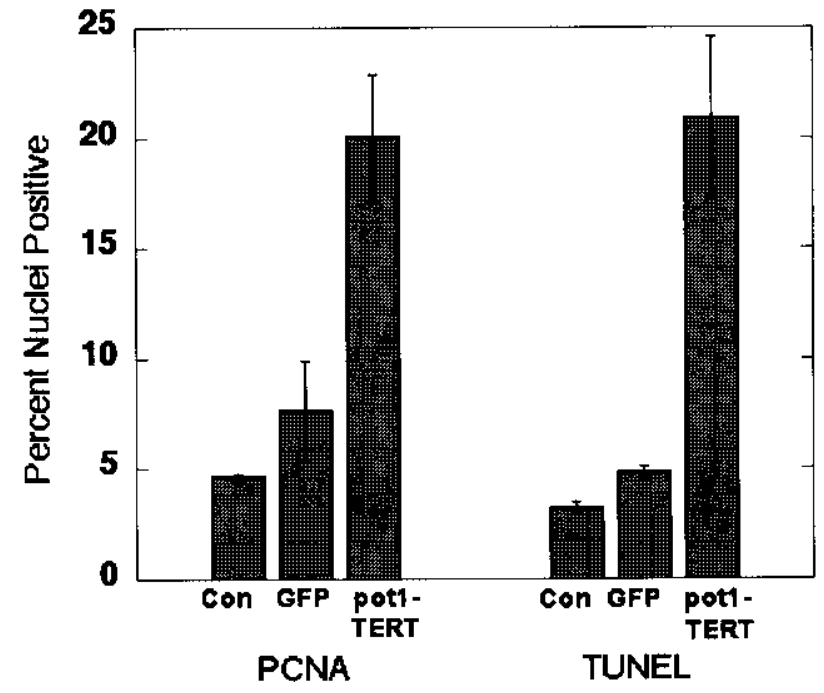

Figure 5. Quantification of PCNA and TUNEL staining. Percentages of positive nuclei for PCNA and TUNEL staining as a fraction of total, counted from two or more slides per vessel. Con: untransduced control SMC; GFP: GFP-transduced SMC; pot1-TERT: pot1-TERT-transduced SMC. Two vessels each were counted for Con, GFP, and pot1-TERT data. No significance is indicated because vessel number is limited. Error bars represent SEMs.

results. The results of the TUNEL staining may be indicative of increased cellular turnover-proliferation as well as cell death and scavenging-in vessels cultured with telomerase-activated cells.

\section{Pot1-TERT SMC Produce More Collagen Than Control Cells in an Engineered Vessel}

Vessels grown using pot1-TERT SMC produced approximately double the collagen compared to vessels grown from control cells or GFP-transduced SMC (Fig. 6). This is in contrast to previous studies, which showed collagen production to be unaffected in TERT-expressing cells compared to control cells (15). This difference may arise from a difference in cell source. Previous work employed cells from 2-year-old donor aorta, whereas this study used cells from the aorta of an adult donor. It is possible that extension of life span translates to maintained collagen production during tissue regeneration from older human cells. This increase in collagen production resulted in an apparent increase in mechanical characteristics. We tested the rupture strength of one pot1-TERT vessel and one vessel grown from control SMC. The pot1-TERT vessel ruptured at $10 \mathrm{psi}$ (roughly $520 \mathrm{mmHg}$ ), while the control vessel ruptured at $5 \mathrm{psi}$.

\section{Implantation of Engineered Vessels}

After 8 weeks of in vitro culture, engineered vessels were implanted into nude rats as aortic interpositional grafts (Fig. 7). There were three experimental groups, with $n=4$ animals in each group: control SMC, GFPtransduced SMC, and pot1-TERT SMC. Because each vessel that was cultured was $8 \mathrm{~cm}$ in length, and because only $1 \mathrm{~cm}$ of length is needed for aortic interposition grafting, we were able to implant multiple aortic grafts from a single engineered vessel. Animals that were implanted with control vessels, using either nontransduced or GFP-transduced cells, all suffered graft failure from rupture or thrombosis and subsequent death within 2-3 $\mathrm{h}$, with most failing immediately after implantation. This was a reflection of the low collagen content and resultant poor mechanical properties of the control SMC and GFP-transduced engineered tissues. In the pot1-TERT group, two animals survived for 1 day and one animal survived for 3 days postimplant. The fourth graft in the pot1-TERT group failed from immediate rupture.

Although preliminary, these findings suggest that vessels engineered using pot1-TERT are superior to those grown with control cells, both from the standpoint of in vitro assessment, and with respect to short-term in vivo function. The benefit of transient telomerase expression appears to be due to an increase in cellular activity together with increased collagen deposition. The coupled findings of increased cellular proliferation as well as apoptosis indicate a cell population undergoing active remodeling, consistent with increased collagen deposition and subsequent superior vessel performance upon implantation. Thus, although preliminary and of

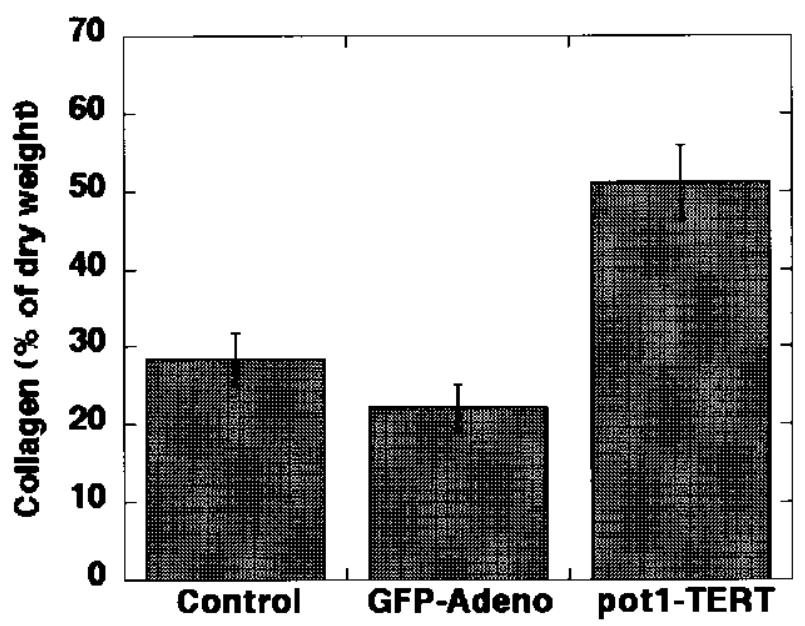

Figure 6. Collagen content of engineered vessels. Collagen content as a fraction of dry weight is shown for vessels cultured from untransduced control cells (Control), from GFPtransduced cells (GFP-Adeno), and from pot1-TERT-transduced cells (pot1-TERT). One vessel each was analyzed for Control and GFP-Adeno data, while two vessels were analyzed for pot1-TERT data. No significance is indicated because vessel number is limited. Error bars represent SEMs. 


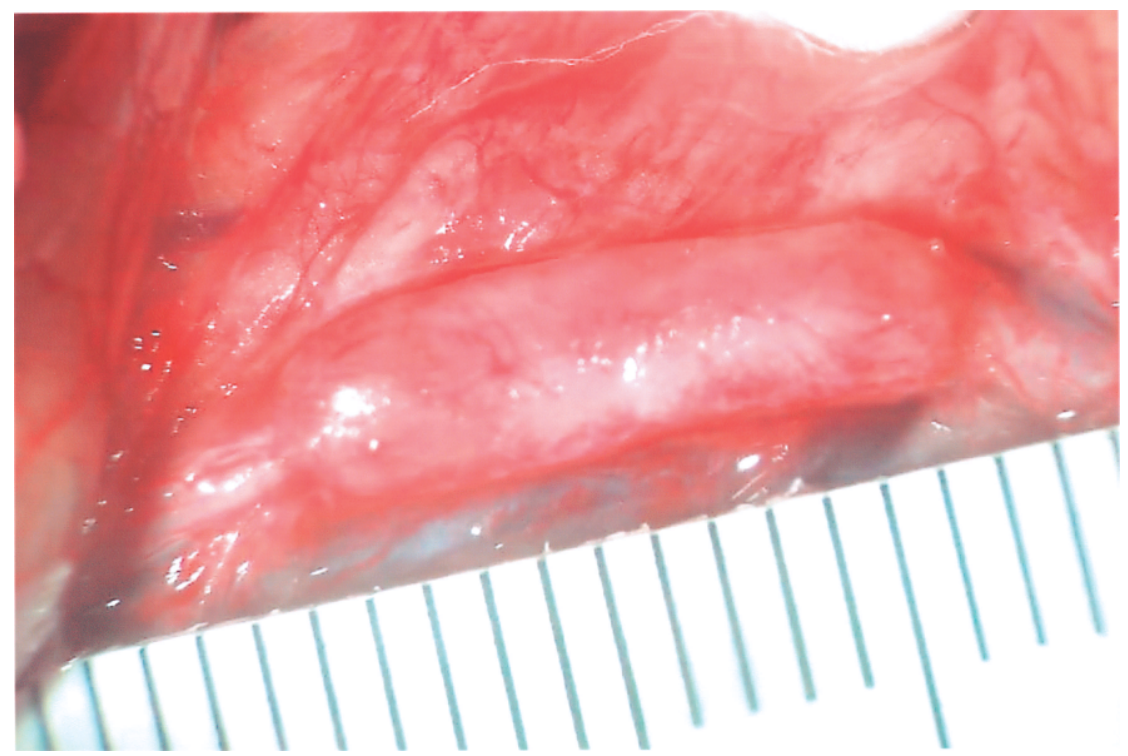

Figure 7. Implantation of human engineered artery. Implantation of 1-mm-diameter pot1-TERTexpressing engineered artery in abdominal aorta of nude rat. Ruler marks indicate mm. Vessel is distended beyond original $1 \mathrm{~mm}$ diameter by physiological pressure.

limited scope, the findings here indicate that transient telomerase activation via pot1-TERT aids in the growth of engineered vessels.

\section{DISCUSSION}

Telomerase activation is necessary to extend cellular life span and allow the growth of engineered vessels from adult smooth muscle cell populations (21). However, telomerase is upregulated in the vast majority of all human malignancies (24). As a result, any tissueengineered product that contains cells with activated telomerase would see challenges to attaining clinical acceptance. In order to overcome these concerns, adenoviral delivery of the fusion protein pot1-TERT was used in these experiments. This approach provides two advantages. First, the pot1-TERT fusion protein provides highly efficient telomerase activity, by facilitating recruitment of telomerase to the telomere via the DNA-binding protein pot1 (1). Second, by delivering pot1-TERT via adenovirus, we were able to activate telomerase only transiently. TRAP analysis confirms that telomerase activity is present in the pot1-TERT cells, but that it declines with higher passages. This decline of telomerase activity with passage is expected and desirable, because the adenovirus is diluted out with cell divisions and because our goal is to have a final cellular product that is devoid of the transgene. While PCR would be required to be absolutely certain the transgene is absent in the cells, adenoviral vectors generally do not to integrate and remain episomal, with an integration rate of only
$10^{-3}$ to $10^{-5}$ per infected cell (9). In conjunction with the fact that these cells were not grown under selection, it is highly unlikely that the transgene remains in high passage cells, which is consistent with the TRAP data. In addition, because of the lack of drug selection pressure during bioreactor vessel culture, cells that had lost adenoviral expression were able to continue replicating along with the cells that still retained adenovirus.

Findings from the TRAP assay are consistent with growth curves, which demonstrate senescence of the control and GFP-transduced cell populations approximately 12 population doublings after transduction, but which show extended replication of the pot1-TERT cultures. The transient telomerase activity in the pot1TERT cells was able to extend the cellular life span of this population as expected. The dual advantage of transient delivery and a highly processive telomerase mutant opens the door to the creation of engineered tissues from adult cell populations in a safe, efficacious manner. It would be insightful for future studies to explore this approach using cells from older donors, who would better represent the target patient population.

From the small number of vessels that could be cultured from a single donor, we found evidence of increased collagen deposition, improved mechanics, and some evidence of improved in vivo function in vessels cultured from pot1-TERT-expressing cells. However, the wall strengths of all of the vessels were not sufficient to reliably support long-term in vivo function, meaning that greater improvement in wall thickness, stiffness, 
and suture retention strength are all still required. Further improvements in matrix deposition, and hence vessel mechanics, may be obtained from optimization of the culture medium to support collagen synthesis from human cells. Such culture medium optimization, examining serum concentration and species, as well as growth factor supplementation, is an ongoing effort.

In this study, we found increased collagen deposition by pot1-TERT cells, presumably due to their increased life span from the transient telomerase activation. This correlates with improved mechanics in the pot1-TERT vessels, and is consistent with previous findings from our laboratory, wherein we used retroviral-mediated delivery of telomerase (hTERT) (21). In our previous reports, we were not able to grow tissues from older (i.e., $>60$ years old) donors using control cells (we were only successful in growing vessels from older donors using hTERT cells), and this skewed our comparisons of pooled vessels grown from control and hTERT cells from multiple donors (21). For hTERT vessels and for control vessels, we have found a correlation between increasing donor age and decreasing collagen content (and vessel mechanics), and also have observed a relative increase of collagen content conferred by hTERT, within specific donors.

Vessels engineered using pot1-TERT SMC contained approximately double the amount of collagen compared to control vessels. This is a promising finding, as collagen is the principal extracellular matrix component responsible for vessel strength $(2,22)$. This correlates with the improved rupture performance, and thus animal survival, seen in aortic implants of the pot1-TERT vessels. Although the animal studies included only a small number of implants, this study indicates that the pot1-TERT vessels appear to provide a functional benefit over vessels cultured from control cells populations. One of the limitations of the vessels implanted in these studies is the lack of an endothelial lining. This is likely the cause of the thrombosis seen within 3 days of implantation in one of the pot1-TERT vessels.

The cells populating the pot1-TERT vessels demonstrate an increased proliferative rate compared to control vessels. We also found an increased rate of apoptosis in the pot1-TERT vessels, which is indicative of overall increased cellular turnover. Other studies on tissue-engineered grafts have noted a coupling of increased proliferative rate and increased apoptosis $(6,14)$, supporting the notion that increased apoptosis per se is not problematic $(11,23)$. Engbers and colleagues found that increased smooth muscle infiltration of an engineered vessel is accompanied by an increased rate of apoptotic markers, supporting the notion that both increased proliferation and increased apoptosis are present in an engineered vessel as the SMCs remodel the scaffold (6). Increased cellular turnover may be closely tied to the observed increased collagen deposition. In analogy to an in vivo example of positive vessel remodeling, Wang and coworkers found increased collagen deposition as well as increased apoptosis in thoracic aortic dissection (26). Taken together, the increased proliferation, increased apoptosis, and increased collagen deposition seen in the pot1-TERT vessels are suggestive of a vessel that is actively remodeling. The ability of the pot1TERT cells to turn over more rapidly and deposit more collagen suggests that transient telomerase activation is a promising approach in the development of an engineered vessel that is more able to adapt to fulfill the demands of in vivo implantation.

\section{CONCLUSION}

We have grown small-diameter tissue-engineered vessels using human smooth muscle cells from an adult donor that were transduced with an adenoviral construct expressing the fusion protein pot1-TERT. The vessels grown from these pot1-TERT cells may offer improved functional performance as aortic interpositional grafts in the nude rat, compared to control vessels. Pot1-TERT vessels demonstrate more proliferating nuclei than control vessels, but also more apoptotic nuclei, indicating higher cell turnover rates. These preliminary findings indicate that transient delivery of telomerase, via the fusion protein pot1-TERT, can allow the growth of improved engineered vessels in a manner that may avoid the safety concerns of long-term telomerase expression.

ACKNOWLEDGMENTS: This work was supported by R21 HL08156 and by R01 HL083895, both to L.E.N. T.H.P is supported by the Medical Scientist Training Program at Duke University (NIH T32 GM007171). L.E.N. has a financial interest in Humacyte, Inc., a regenerative medicine company. Humacyte did not fund these studies, and Humacyte did not affect the design, interpretation, or reporting of any of the experiments herein.

\section{REFERENCES}

1. Armbruster, B. N.; Linardic, C. M.; Veldman, T.; Bansal, N. P.; Downie, D. L.; Counter, C. M. Rescue of an hTERT mutant defective in telomere elongation by fusion with hPot1. Mol. Cell. Biol. 24:3552-3561; 2004.

2. Armentano, R. L.; Bara, J. G.; Levenson, J.; Simon, A.; Pichel, R. H. Arterial wall mechanics in conscious dogs. Circ. Res. 76:468-478; 1995.

3. Baumann, P.; Cech, T. R. Pot1, the putative telomere endbinding protein in fission yeast and humans. Science 292: 1171-1175; 2001.

4. Bodnar, A. G.; Ouellette, M.; Frolkis, M.; Holt, S. E.; Chiu, C. P.; Morin, G. B.; Harley, C. B.; Shay, J. W.; Lichtsteiner, S.; Wright, W. E. Extension of life-span by introduction of telomerase into normal human cells. Science 279:349-352; 1998.

5. Colgin, L. M.; Baran, K.; Baumann, P.; Cech, T. R.; Reddel, R. R. Human POT1 facilitates telomere elongation by telomerase. Curr. Biol. 13:942-946; 2003.

6. Engbers-Buijtenhuijs, P.; Buttafoco, L.; Poot, A. A.; Dijkstra, P. J.; de Vos, R. A. I.; Sterk, L. M. Th.; Geelkerken, 
R. H.; Vermes, I.; Feijen, J. Biological characterization of vascular grafts cultured in a bioreactor. Biomaterials 27: 2390-2397; 2006.

7. Grant, R. A. Estimation of OH-proline by the autoanalyser. J. Clin. Pathol. 17:685-686; 1964.

8. Hacein-Bey-Abina, S.; Garrigue, A.; Wang, G. P.; Soulier, J.; Lim, A.; Morillon, E.; Clappier, E.; Caccavelli, L.; Delabesse, E.; Beldjord, K.; Asnafi, V.; MacIntyre, E.; Dal Cortivo, L.; Radford, I.; Brousse, N.; Sigaux, F.; Moshous, D.; Hauer, J.; Borkhardt, A.; Belohradsky, B. H.; Wintergerst, U.; Velez, M. C.; Leiva, L.; Sorensen, R.; Wulffraat, N.; Blanche, S.; Bushman, F. D.; Fischer, A.; Cavazzana-Calvo, M. Insertional oncogenesis in 4 patients after retrovirus-mediated gene therapy of SCID-X1. J. Clin. Invest. 118:3132-3142; 2008.

9. Harui, A.; Suzuki, S.; Kochanek, S.; Mitani, K. Frequency and stability of chromosomal integration of adenovirus vectors. J. Virol. 73:6141-6146; 1999.

10. Klinger, R. Y.; Blum, J. L.; Hearn, B.; Lebow, B.; Niklason, L. E. Relevance and safety of telomerase for human tissue engineering. Proc. Natl. Acad. Sci. USA 103:25002505; 2006.

11. Kockx, M. M.; Herman, A. G. Apoptosis in atherosclerosis: Beneficial or detrimental? Cardiovasc. Res. 45:736746; 2000.

12. Lei, M.; Zaug, A. J.; Podell, E. R.; Cech, T. R. Switching human telomerase on and off with hPot1 protein in vitro. J. Biol. Chem. 280:20449-20456; 2005.

13. Loayza, D.; de Lange, T. POT1 as a terminal transducer of TRF1 telomere length control. Nature 423:1013-1018; 2003.

14. Mayr, M.; Li, C.; Zou, Y.; Huemer, U.; Hu, Y.; Xu, Q. Biomechanical stress-induced apoptosis in vein grafts involves p38 mitogen-activated protein kinases. FASEB J. 15:261-270; 2000.

15. McKee, J. A.; Banik, S. R.; Boyer, M. J.; Hamad, N. M.; Lawson, J. H.; Niklason, L. E.; Counter, C. M. Human arteries engineered in vitro. EMBO Rep. 4:633-638; 2003.
16. Minamino, T.; Miyauchi, H.; Yoshida, T.; Tateno, K.; Kunieda, T.; Komura, I. Vascular cell senescence and vascular aging. J. Mol. Cell. Cardiol. 36:175-183; 2004.

17. Miyoshi, T.; Kanoh, J.; Saito, M.; Ishikawa, F. Fission yeast Pot1-Tpp1 protects telomeres and regulates telomere length. Science 320:1341-1344; 2008.

18. Mukherjee, D.; Bhatt, D. L.; Roe, M. T.; Patel, V.; Ellis, S. G. Direct myocardial revascularization and angiogenesis-how many patients might be eligible? Am. J. Cardiol. 84:598-600; 1999.

19. Niklason, L. E.; Gao, J.; Abbott, W. M.; Hirschi, K.; Houser, S.; Marini, R.; Langer, R. Functional arteries grown in vitro. Science 284:489-493; 1999.

20. Nolan, K.; Millet, Y.; Ricordi, C.; Stabler, C. L. Tissue engineering and biomaterials in regenerative medicine. Cell Transplant. 17:241-243; 2008.

21. Poh, M.; Boyer, M.; Solan, A.; Dahl, S.; Pedrotty, D.; Banik, S.; McKee, J. A.; Klinger, R.; Counter, C. M.; Niklason, L. E. Blood vessels engineered from human cells. Lancet 365:2122-2124; 2005.

22. Roach, M. R.; Burton, A. C. The reason for the shape of the distensibility curves of arteries. Can. J. Biochem. Physiol. 35:681; 1957.

23. Rossig, L.; Dimmeler, S.; Zeiher, A. M. Apoptosis in the vascular wall and atherosclerosis. Basic Res. Cardiol. 96: $11-22 ; 2001$

24. Shay, J. W.; Bacchetti, S. A survey of telomerase activity in human cancer. Eur. J. Cancer 33:787-791; 1997.

25. Tsuruga, Y.; Kiyono, T.; Matsushita, M.; Takahashi, T.; Kasai, H.; Matsumoto, S.; Todo, S. Establishment of immortalized human hepatocytes by introduction of HPV16 E6/E7 and hTERT as cell sources for liver cell-based therapy. Cell Transplant. 17:1083-1094; 2008.

26. Wang, X.; LeMaire, S. A.; Chen, L.; Shen, Y. H.; Gan, Y.; Bartsch, H.; Carter, S. A.; Utama, B.; Ou, H.; Coselli, J. S.; Wang, X. L. Increased collagen deposition and elevated expression of connective tissue growth factor in human thoracic aortic dissection. Circulation 114(I):200205; 2006. 
